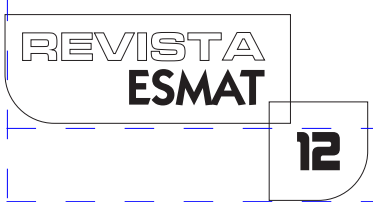

\title{
LA NOTION DE TORTURE, DE TRAITEMENTS INHUMAINS ET DEGRADANTS DANS LA JURISPRUDENCE DE LA COUR EUROPEENNE DES DROITS DE L'HOMME
}

THE NOTION OF TORTURE AND UNHUMAN AND HUMILIATING TREATMENTS FROM THE JURISPRUDENCE OF THE HUMAN RIGHTS EUROPEAN COURT

Jean-Paul CÉRÉ

Responsable de l'Equipe de Pau (Institut de sciences criminelles et de la Justice de l'Université Bordeaux). Président de l'Association Française de Droit Pénal. Président du Comité International des Pénalistes Francophones. www.jpcere.fr

\section{RESUME}

En vertu de l'article 3 de la Convention européenne de sauvegarde des droits de I'homme, les Etats ne peuvent ni pratiquer la torture ni infliger des traitements inhumains ou dégradants. Le particularisme de cette convention par rapport aux autres textes internationaux ou régionaux relatifs aux droits de l'homme réside indéniablement dans le système de protection existant qu'elle a organisé. Un organe juridictionnel, la cour européenne des droits de l'homme, est en charge de sanctionner les Etats qui ne respectent la Convention. Au terme d'une jurisprudence novatrice et évolutive, la cour européenne des droits de l'homme impose de plus en plus efficacement de lutter contre la torture, les traitements inhumains et dégradants.

MOTS-CLES: Droits de l'homme ; Torture ; Système de protection.

\section{ABSTRACT}

By virtue of the article 3 of the European convention of protection of human rights, States cannot either practise the torture or impose inhuman or degrading treatments. The sense of identity of this convention with regard to the other international or regional texts relative to human rights lives unmistakably in the existing system of protection which she organized. A jurisdictional organ, the European Court of Human Rights, handles to punish the States which respect the Convention. In the term of an innovative and evolutionary jurisprudence, the European Court of Human Rights imposes more and more effectively to fight against the torture, the inhuman and degrading treatments.

KEYWORDS : Human rights; Torture ; System of protection. 


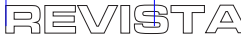

ESMAT

JEAN-PAUL CÉRÉ

\section{INTRODUCTION}

La protection européenne des droits de l'homme trouve ses origines dans les avancés diplomatiques de l'après deuxième guerre mondiale. A cette époque les pays industrialisés ont cherché à promouvoir les droits de l'homme et les libertés fondamentales. René CASSIN projetait, quant à lui, l'objectif de « protéger tout l'homme et protéger les droits de tous les hommes ».

C'est en fait la création du conseil de l'Europe qui va bouleverser l'approche de la protection supra nationale des droits de l'homme. Les statuts du conseil de l'Europe sont adoptés à Londres le 5 mai 1949. Les divers États signataires marquent leur attachement au respect des droits de l'homme et des libertés fondamentales. L'article 3 du statut indique ainsi que «tout membre du Conseil de l'Europe reconnaît le principe de la prééminence du droit et le principe en vertu duquel toute personne placée sous sa juridiction doit jouir des droits de l'homme et des libertés fondamentales ». Cette prééminence du droit est, d'après les statuts même du Conseil de l'Europe, le signe est le signe d'une démocratie véritable.

Très rapidement après, une première convention conclu sous l'égide du Conseil de l'Europe naît. II s'agit de la Convention européenne des droits de l'homme du 4 novembre 1950 qui entre en vigueur le 3 septembre 1953. Cette convention amplifie les bases statutaires du Conseil de l'Europe. Son préambule souligne l'attachement des États parties au « patrimoine commun » des États membres, c'est à dire leur attachement aux valeurs morales et spirituelles qui sont à l'origine des principes de liberté individuelle, de liberté politique et de prééminence du droit. II réaffirme que le maintien de la justice et de la paix repose «sur un régime politique véritablement démocratique » et sur « un commun respect des droits de l'homme ».

Le succès du Conseil de l'Europe est réel. 47 pays en font partis. La plupart des pays de l'Europe de l'est et de l'Europe centrale sont membres. La Russie a adhéré le 28 février 1996. L'adhésion au Conseil de l'Europe implique de ratifier rapidement la Convention européenne des droits de l'homme car celle-ci représente une garantie efficace du respect des droits de l'homme. II faut savoir que la Convention européenne des droits de l'homme comprend une quinzaine de protocoles additionnels (ex. art. 2 du protocole 7 prévoit le double degré de juridiction en matière répressive) qui présentent une force obligatoire identique à la convention elle-même.

Le particularisme de la convention par rapport aux autres textes internationaux ou régionaux relatifs aux droits de l'homme réside indéniablement dans le système de protection existant. Elle comprend un organe politique de décision (le conseil des ministres) et surtout un organe juridictionnel de protection (la cour européenne des droits de l'homme qui a son siège à Strasbourg).

Depuis les années 50 de nombreuses autres conventions ont été adoptés par le conseil de l'Europe dans des domaines des plus divers (ex. convention sur le transfèrement des détenus). 
La Convention européenne de sauvegarde des droits de l'homme est véritablement source de garanties supplémentaires par comparaison des autres textes supra nationaux, en raison du mécanisme de contrôle des droits qu'elle protège. De caractère impératif, elle aménage un recours pour violation de l'une de ses dispositions devant la Cour européenne des droits de l'homme. Ratifiée par le Parlement en décembre 1974, la France a admis les recours individuels à compter du 9 octobre 1981. Depuis l'entrée en vigueur du protocole I I le ler novembre 1998 (en 2000), la cour européenne des droits de l'homme est devenue une cour et permanente. Auparavant, la Cour n'était pas une juridiction permanente.

Les mauvais traitements sont prohibés par la Convention européenne de sauvegarde des droits de l'homme en termes absolus. Ils le sont également par la convention européenne pour la prévention contre la torture, les peines et traitements inhumains et dégradants (entrée en vigueur le lerfévrier 2007).

Ils ne doivent pas subir de restrictions ou de dérogations quelconques. La jurisprudence et la doctrine considèrent en effet ce droit comme l'une des valeurs fondamentales de toute société démocratique.

En vertu de l'article 3 Convention européenne des droits de l'homme, les Etats ne peuvent ni pratiquer la torture ni infliger des traitements inhumains ou dégradants. Cette exigence textuelle est amplifiée par une autre obligation qui découle de la jurisprudence en vertu de laquelle les états doivent aussi protéger toute personne relevant de leur juridiction quand bien même la situation de danger s'accomplit en dehors de celle-ci.

II importe peu que les traitements proviennent de personnes appartenant à la fonction publique. Si le danger est le fait de particuliers ou de groupes de particuliers, l'article 3 peut quand même trouver à s'appliquer. C'est le caractère absolu de la protection de cet article qui commande une telle solution. Dès lors les Etats doivent prendre toutes les dispositions afin d'empêcher que des personnes placées sous leur juridiction subissent des mauvais traitements, même s'ils sont infligés par des particuliers. L'article 3 s'applique aux relations interindividuelles. ${ }^{2}$ La cour fait donc une application aussi bien verticale de l'article 3 (actes commis par des agents de l'Etat), qu'horizontale (actes commis par des particuliers ou par la victime elle-même). L'interprétation de la notion de torture, de traitements inhumains et dégradants est interprétée de façon large par la cour européenne des droits de l'homme. Tant, la définition de la notion (I), que l'application jurisprudentielle qui en résulter ne laissent guère de doutes (II).

\footnotetext{
'CEDH7 juill. 1989, Soering c/RU

${ }^{2}$ Par ex. châtiments corporels infligés à un enfant par son beau père, la cour a déclaré que l'article 3 s'appliquait bien, CEDH 23 sept. 1998, A. c/RU
} 


\section{La définition des traitements interdits \\ A. L'exigence d'un seuil de gravité des souffrances infligées \\ I. Une interprétation relative du seuil de gravité}

L'article 3 de la Convention européenne des droits de l'homme dispose que « nul ne peut être soumis à la torture ni à des peines ou traitements inhumains et dégradants ». Pour la Cour européenne, " un mauvais traitement doit atteindre un minimum de gravité » pour que l'article 3 s'applique. Elle se positionne, en somme, par référence à un critère d'intensité des souffrances infligées. Dès lors elle a pu juger, par exemple, qu'un traitement « désagréable » se situait en deçà du seuil requis ${ }^{3}$, alors que le « syndrome du couloir de la mort » dépassait la limite fixée et emportait l'application de l'article 3. Le critère de l'intensité des souffrances se retrouve dans l'ensemble des décisions rendues parla Cour.

La fixation du seuil de référence qui offre ou non la protection de l'article $3{ }^{4}$ est, de l'aveu même de la cour, « relative par essence ». L'appréciation « dépend de l'ensemble des données de la cause, notamment de la durée du traitement et de ses effets physiques et mentaux, ainsi que parfois, du sexe, de l'âge, de l'état de santé de la victime... ». Cela signifie, en d'autres termes, qu'elle se réfère à la situation personnelle de la victime, mais pas exclusivement. La Cour tend aussi à intégrer des paramètres sociologiques ou socio-politiques, de manière à ménager « un juste équilibre » entre l'intérêt général et celui de l'individu.

La cour, notamment à propose de l'article 3 interprète la Convention de façon évolutive. Elle l'interprète « à la lumière des conditions d'aujourd'hui » ${ }^{5}$. L'affaire Selmouni c/ France qui concerne directement l'article 3 est une parfaite illustration. ${ }^{6}$ La cour adopte un principe similaire par lequel elle retient la qualification de torture contre la France au motif que "le niveau d'exigence croissant en matière de protection des droits de I'homme et des libertés fondamentales implique parallèlement et inéluctablement une plus grande fermeté dans l'appréciation des atteintes aux valeurs fondamentales des sociétés démocratiques ». Cela veut dire que la Cour élève le niveau d'exigence relativement à l'article 3 .

La jurisprudence de la cour s'est orientée vers une approche qui semble très sérieusement à minimiser l'existence même du seuil de gravité. Elle a affirmé dans certains arrêts que toute brutalité, quelque soit sa gravité est contraire à l'article $3^{7}$. Cette

\footnotetext{
${ }^{3} \mathrm{CEDH} 6$ nov. 1980, Guzzardi / Italie

${ }^{4} \mathrm{CEDH} 7$ juill. 1989, Soering c/ Royaume-Uni

${ }^{5} \mathrm{CEDH}$ Marckxc/Belgique, I 3 juin 1979

${ }^{6}$ Là où plusieurs années auparavant, elle aurait pu considérer les faits reprochés comme des traitements inhumains, CEDH 28 juill. 1999, Selmouni c/France

${ }^{7}$ V. CEDH 27 août I 992, Tomasi c/ France
} 
jurisprudence confère un caractère absolu au principe d'intégrité physique de la personne. Elle a été dégagée dans un premier temps lorsque la personne qui avait subis les mauvais traitements était privée de sa liberté. Elle revient à considérer que tout usage de la force contre une personne privée de sa liberté est intolérable dans une société démocratique.

En réalité le degré minimum de gravité n'a pas réellement disparu dans la jurisprudence de la cour. II faut déduire de cette jurisprudence qu'il existe une sorte de présomption de gravité de tout usage de la force physique sur une personne privée de sa liberté. Tout usage de la force physique sur une personne privée de liberté atteint, de fait, le minimum de gravité exigé pour constituer une violation de l'article 3. En toute hypothèse, si la force est utilisée, elle doit être à la fois nécessaire et proportionnée. $A$ défaut, il y aura violation de l'article 3 (ex. traitement inhumain et dégradant pour un usage disproportionné de la force sur un mineur lors d'une vérification d'identité dans un commissariat $^{8}$.

Cette jurisprudence s'applique maintenant aussi en matière d'usage de la force lors d'arrestation. Pour la cour européenne, l'article 3 n'interdit pas le recours à la force afin de procéder à une arrestation, mais un tel recours ne doit intervenir que lorsqu'il est strictement nécessaire et proportionné. ${ }^{9} \mid l$ en va de même à fortiori en cas de résistance « passive ${ }^{10}$.

L'article 3 est violé en cas d'usage excessif de la force " , sans considération de lieu ${ }^{12}$

${ }^{8} \mathrm{CEDH} 4$ nov. 20 I0, Darraj c/ France (mineur de 16 ans est emmené au commissariat de police pour un contrôle d'identité et en ressort, deux heures plus tard, avec de multiples blessures occasionnant 21 jours d'ITT, dont une fracture testiculaire ; il indique avoir essuyé coups et insultes après avoir refusé d'être menotté. Les policiers, de leur côté, disent avoir dû le plaquer au sol pour le maitriser et donner des coups de genoux pour se protéger).

${ }^{9} \mathrm{CEDH} 23$ juill. 2013, İzci c. Turquie (requérante qui n'oppose aucune résistance et ces derniers utilisent des gaz lacrymogènes).

${ }^{10} \mathrm{CEDH} 3$ oct. 2013, Douet c. France (incapacité totale de travail de cinq jours alors que le requérant se contentait de résister passivement à son arrestation en refusant de sortir de son véhicule et de mettre ses mains derrière le dos pour être menotté).

"CEDH 24 janv. 20 I2, lodan Petrov c. Bulgarie, n²2926/04 (surveillants équipés de matraques et absence de mention de blessures qu'auraient subis les surveillants lors de leur affrontement avec le détenu dont des certificats médicaux faisaient état pour ce dernier d'ecchymoses et contusions). CEDH 2 oct. 20 I2, Jurijs Dmitrijevs c. Lettonie, $n^{\circ} 37467$ (passage à tabac par des surveillants et violences par des agents d'une unité spéciale d'intervention). Une solution identique prévaut lors de l'arrestation de l'individu, CEDH 28 nov. 2000, Rehbock c. Slovénie, n²9462/95 (personne arrêté et matraqué alors que les policiers étaient plus nombreux que les suspects); $\mathrm{CEDH} 26$ avr. 2016, Seagal c. Chypre, n 50756/13 (détenu présentant plusieurs blessures à la suite d'interventions de surveillants et d'autres détenus)

${ }^{12}$ Pour une violation lors d'un transfert d'un détenu, CEDH 03 avr. 2012, Dimitar Dimitrov c. Bulgarie, $n^{\circ}$ 18059/05. 
ou de circonstances. ${ }^{13}$ Dès lors que l'utilisation de la force physique n'est pas rendu nécessaire par le comportement de la personne, la violation de l'article 3 est admise. En revanche, en cas de comportement violent et avéré, c'est à cette dernière de le contredire pour pouvoir se prévaloir avec succès de l'article $3^{14}$.

Certains moyens utilisés en détention utilisés contre des détenus agressifs ou refusant d'obéir aux ordres des personnels pénitentiaires sont considérés aussi par la Cour comme illégitimes. Tel est le cas du gaz poivre dans une cellule ${ }^{15}$ ou encore l'immobilisation du détenu sur un lit de contentieux. Cette dernière mesure ne peut être utilisée pour la cour pour punir un détenu mais seulement pour les empêcher d'agir d'une manière dangereuse contre lui-même ${ }^{16}$.

Cette application relative du seuil de gravité permet de faire entrer dans le champ d'application de l'article 3 d'autres mesures. Ainsi, la jurisprudence de la cour permet de considérer que « dès lors qu'ils ont fait l'objet d'une décision les expulsions, extraditions ou refoulements, qu'ils soient effectifs ou virtuels, sont susceptibles de constituer des traitements inhumains ou dégradants.

Le risque invoqué n'est pas limité, de surcroît, aux seuls actes des autorités publiques mais s'étend aux personnes ou groupes de personnes qui ne relèvent pas de la fonction publique. Elle juge toutefois qu'une extradition vers un pays où les requérants risquaient une condamnation à la réclusion criminelle à perpétuité pour meurtre ne violait pas leurs droits fondamentaux protégés par l'article $3^{17}$.

On peut relever aussi que le franchissement de ce seuil dépasse la personne qui subi des traitements contraires à la convention. L'article 3 peut être invoqué par des proches des détenus. Ainsi, la cour a pu retenir que la mère d'un détenu avait fait l'objet d'un traitement inhumain et dégradant comme conséquence du décès de son fils ${ }^{18}$.

${ }^{13}$ CEDH 6 nov. 2012, Borodin c. Russie, n $n^{\circ} 4$ 1867/04 (détenu qui reçoit un coup des coups de matraques de la part d'un surveillant lors d'une fouille alors qu'il n'est pas démontré qu'il avait un comportement particulièrement dangereux); CEDH I 7 janv. 20 I3, Karabet et autres c. Ukraine, $n^{\circ}$ 38906/07 et 52025/07 (usage de la force visant à écraser un mouvement de grève de la faim)

${ }^{14}$ CEDH 7 juill. 20 I I, Hellig c. Allemagne, $n^{\circ}$ 20999/05 (comportement à risque du détenu constaté par les juridictions internes qui ont relevé que ce dernier avait usé de violence contre les surveillants et que les lésions qu'ils dénonçaient présentaient un caractère mineur, à savoir ecchymoses sans gravité et un hématome pouvant guérir sans complications, selon un rapport médical);

${ }^{15} \mathrm{CEDH}$ I 3 févr. 2014, Talic. Estonie, $\mathrm{n}^{\circ}$ 66393/I 0.

${ }^{16} \mathrm{CEDH}$ I 3 févr. 20 I 4, Tali c. Estonie, $\mathrm{n}^{\circ}$ 66393/I 0 (immobilisation durant trois heures et demie); CEDH 29 mai 2012, Julin c. Estonie, $n^{\circ}$ 16563/08, 4084I/08, 8192/08 et 18656/10 (immobilisation durant neuf heures) 


\section{La distinction des traitements interdits}

C'est en appliquant la notion de seuil de gravité que la cour parvient à distinguer et à définir les différentes notions interdites par l'article 3, à savoir la torture, les traitements inhumains ou dégradants.

La Cour détermine la nature des agissements contestés au sens de l'article 3. Leur jurisprudence permet de distinguer les notions de torture, de traitements inhumains et de peines ou traitements dégradants selon une hiérarchie décroissante. Elle sousentend que « toute torture ne peut être qu'un traitement inhumain et dégradant et tout traitement inhumain [...] ne peut être que dégradant ».

Pour opérer une différentiation, entre la torture et les autres traitements, la jurisprudence se réfère à l'intensité des souffrances infligées. C'est le critère majeur de distinction. Les souffrances infligées doivent déboucher sur « des traitements inhumains délibérés provoquant de fort graves et cruelles souffrances $"{ }^{19}$.

Le concept de torture n'est pas réducteur aux seules violences physiques et englobe les souffrances mentales ou morales d'une particulière gravité. Au sens de la jurisprudence de la Cour de Strasbourg, comme d'après l'article I de la Convention des Nations Unies sur la torture et autres traitements cruels, inhumains ou dégradants, entrée en vigueur le 26 juin 1987, la torture revêt trois éléments : "intensité des souffrances, intention délibérée, but déterminé ». La torture ne diffère donc pas, par nature, des traitements inhumains. Elle en est une forme aggravée ${ }^{20}$.

Les traitements inhumains se définissent comme ceux qui provoquent volontairement de graves souffrances mentales ou physiques qui ne peuvent se justifier. "La souffrance provoquée doit se situer à un niveau particulier pour que l'on puisse qualifier une peine d'inhumaine au sens de l'article 3 ».

Ainsi, le fait de détenir un enfant de cinq ans pendant deux mois dans un centre de rétention initialement conçu pour adultes est constitutif d'un traitement inhumain ${ }^{21}$. II s'agissait de ressortissants congolais en situation irrégulière en Belgique qui avait été détenue, pour ce qui concerne l'enfant, dans un lieu de détention inadapté tout simplement parce qu'il n'existait pas à l'époque de structures adéquates. La cour

${ }^{17} \mathrm{CEDH} 3$ juill. $200 \mathrm{I}$, Nivette c. France

${ }^{18} \mathrm{CEDH}$ I 4 mars 20 I3, Salakhov et Islyamova c. Ukraine, n² 28005/08

${ }^{19} \mathrm{CEDH}$ I 8 janv. 1978, Irlande c/Royaume-Uni

${ }^{20}$ Par ex. CEDH 5 juin 20 I2, Buntov c. Russie, $n^{\circ} 27026 / 10$ (gardiens de prison qui arrachent tous les ongles des pieds et des mains d'une détenu avec des tenailles, tentent de l'empoisonner, alors même que l'intéressé est détenu et transporté dans des conditions difficiles). 
considère que l'intéressé se trouvait dans une situation d'extrême vulnérabilité, que la détention l'avait placé dans un état de profond désarroi, et que cette mesure pouvait emporter des conséquences psychologiques graves.

Parfois, elle utilise le terme de peine inhumaine. Cette qualification peut s'expliquer par la prise en compte de l'exécution de la mesure et non pas de son seul prononcé. II en va ainsi de la punition de 350 flexions infligées à un soldat car il n'avait pas correctement nettoyé les baraquements. II s'agissait d'un appelé, dispensé d'exercices physiques en raison de douleurs récurrentes au genou. II s'était effondré durant la punition. II avait été conduit à l'hôpital, où les médecins avaient diagnostiqué une lésion invalidante de la colonne vertébrale ${ }^{22}$.

Les traitements dégradants reviennent quant à eux à « créer chez les personnes des sentiments de peur, d'angoisse et d'infériorité, propres à les humilier, à les avilir et à briser éventuellement leur résistance physique et morale » ou poussent l'individu «à agir contre sa volonté ou sa conscience. Ainsi, par exemple, le fait d'exposer un individu lors de son procès dans une cage comprenant des barreaux en métal est un traitement dégradant (humilie le requérant à ses propres yeux ${ }^{23}$. Une gifle infligée par des agents des forces de l'ordre à des personnes qui se trouvent sous leur contrôle est un traitement dégradant ${ }^{24}$.

Un arrêt est également intéressant sur cette notion de traitement dégradant. II reflète en outre l'extension récente du champ d'application de la jurisprudence de l'article 3 est d'une protection de plus en plus large ${ }^{25}$. La cour dans cette affaire considère comme contraires à l'article trois, l'administration de force de vomitifs à un individu soupçonné de trafic de stupéfiants qui avait tenté de détruire les preuves de son comportement délictueux. Elle constate que les autorités l'ont forcé à vomir, non pas pour des raisons thérapeutiques mais pour recueillir des éléments de preuve qu'elles auraient également pu obtenir par des méthodes moins intrusives. Selon une formule classique elle en conclut que la mesure " était de nature à inspirer au requérant des sentiments de peur, d'angoisse et d'infériorité propre à l'humilier et à l'avilir ». II faut en outre noter, cela n'a pas échappé à la cour, que la mesure comportait des risques pour la santé de l'intéressé, en particulier en raison du manquement à procéder préalablement à une anamnèse adéquate (examens, interrogatoire sur l'histoire du sujet).

En somme les peines ou traitements inhumain concernent l'intégrité physique de la personne car lésions et souffrances physiques tandis que les peines ou traitements

${ }^{21}$ CEDH 12 oct. 2006, Mubilanzila et autres c/Belgique; CEDH 19 jav. 20 I2, Popov c/France;

${ }^{22} \mathrm{CEDH} 12$ juill. 2016 , A. B. et autres c/ France (5 arrêts).

${ }^{23} \mathrm{CEDH} 3$ juill. 2008, Chember c/Russie

${ }^{24} \mathrm{CEDH} 28$ sept. 2015 , Bouyid c. Belgique

${ }^{25} \mathrm{CEDH}$ Gde ch. I I juill. 2006, Jalloh c/Allemagne 
dégradants intéressent la dignité de la personne au regard de la peur, de l'angoisse et de l'avilissement qu'ils provoquent ${ }^{26}$.

\section{B. Les aspects procéduraux I. La preuve des traitements interdits}

Sur le terrain de la preuve des mauvais traitements, la jurisprudence a notablement évoluée dans un sens très favorable pour les victimes de mauvais traitements. Elle trouve à s'appliquer en premier lieu à toutes les personnes privées de liberté. La Cour intègre la condition de particulière vulnérabilité des personnes privées de liberté pour décider qu'il appartient au gouvernement défendeur de démontrer que les allégations de sévices subis pendant une détention ne sont pas avérés ${ }^{27}$.

Pour une garde à vue, la cour considère que si l'individu est en bonne santé « et que I'on constate qu'il est blessé au moment de sa libération, il incombe à l'Etat de fournir une explication plausible pour l'origine de ses blessures, à défaut de quoi l'article 3 de la convention trouve à s'appliquer " ${ }^{28}$.

Dans l'hypothèse où la victime ne peut produire une preuve médicale et même s'il n'existe pas de traces visibles de blessures, la démarche du requérant ne perd pas toutes chances d'aboutir (elle est juste affaiblie). L'absence de telles preuves n'est pas systématiquement « de nature à saper la crédibilité des allégations du requérant en général » ${ }^{29}$.

II s'agit donc d'une authentique présomption de causalité qui jouera chaque fois que la preuve n'est pas établie " au delà de tout doute raisonnable, sachant qu'une telle preuve peut résulter d'un faisceau d'indices suffisamment graves, précis et concordants » représenté par exemple par la présence de plusieurs certificats médicaux suffisamment éclairants ou par « l'absence d'explications plausibles pour l'origine des blessures ». Au surplus, la charge de la preuve des mauvais traitements incombe à l'Etat. II est tenu de s'expliquer sur l'origine des blessures ou des lésions présentées par la victime et celle-ci n'a pas ainsi à démontrer l'existence d'un lien entre ses traumatismes et les conditions dans lesquelles elle a été détenue. Pour établir la véracité des faits pertinents, la Cour

\footnotetext{
${ }^{26}$ V. F. Tulkens, Droits de l'homme et prison in J.P. Céré (dir.), Panorama européen de la prison, L'Harmattan, 2002, p. 35.

${ }^{27}$ CEDH Klaas c/Allemagne, 22 sept. 1993

${ }^{28} \mathrm{CEDH}$ Selmouni c/ France op. cit.; CEDH 16 juill. 2015, Ghedir et autres contre France (interpellation brutale (coups de genoux dans le visage) puis placement en garde à vue, le requérant plonge dans le coma dès le début du placement en garde à vue qui l'entraîne, quelques années plus tard, à une incapacité quasiment permanente).

${ }^{29} \mathrm{CourEDH}, 9$ juin 1998, Tekin c/Turquie
} 
s'appuie sur l'ensemble des éléments de preuve fournis par les parties ou qu'elle s'est, au besoin, procurés d'office.

\section{Les obligations d'enquêtes}

Toutes les fois où une violation de l'article 3 de la convention paraît plausible, l'Etat défendeur est tenu de procéder à une enquête officielle en vue identifier et de punir les responsables des mauvais traitements. L'obligation revient en somme à proposer à la victime prétendue un « recours effectif » au sens de l'article I 3 de la Convention.

II ne suffit pas de déclencher une enquête, des poursuites pénales, voire même de parvenir à une condamnation pour obtenir un constat de conventionalité. II doit s'agir d'une enquête véritable, qui doit conduire à la manifestation de la vérité. Les autorités doivent mettre en œuvre des diligences raisonnables pour parvenir à cette fin. Le manquement à cette exigence de diligences raisonnables entraîne la violation de l'article 3 dans son volet procédural. Ainsi nonobstant une enquête et des poursuites pénales exercées contre les auteurs des mauvais traitements, la cour peut conclure une violation de l'article $3^{30}$.

$=>$ il s'agissait d'un enfant de 12 ans qui travaillait comme apprenti et qui avait dérobé 275 \$ environ à son employeur. Son père s'étant arrangé avec ce dernier, la plainte fut retirée. Le garçon avait été placé en garde à vue, il fut de ce fait libéré et le père dut signer une déclaration selon laquelle son fils avait été bien traité au commissariat, qui est en bonne santé et qu'il ne souhaitait pas qu'il soit examiné par un médecin. Ses parents se rendirent compte ensuite qu'il avait des blessures et des hématomes sur le corps. Le père déposa ensuite plainte, les policiers furent poursuivis, est jugé par une cour d'assises qui reconnu que l'enfant avait été battu par les policiers. Après un pourvoi devant la Cour de Cassation, qui qualifia les faits en délit d'extorsion d'aveu, la cour d'assises de renvoi infligea la peine minimum à savoir un an de réclusion. Dans cette affaire cette peine n'empêcha pas les policiers en cause d'être promus à des grades supérieurs.

Pour la cour « le système pénal, tel qu'il a été appliqué en l'espèce, s'est avéré loin d'être rigoureux et ne pouvait engendrer aucune force dissuasive susceptible d'assurer la prévention efficace d'actes illégaux tels que ceux dénoncés par le requérant. La cour conclut dès lors que l'issue de la procédure pénale litigieuse n'a pas offert un redressement approprié de l'atteinte portée à la valeur consacrée dans l'article 3 ».

Evidemment si la personne nécessite des soins (ex. personne blessée) durant la garde à vue, ceux-ci doivent être assurés. La cour a même spécifiée qu'une personne ne doit pas être ramenée trop précipitamment en garde à vue, après une intervention chirurgicale. Elle fait primer ce faisant le suivi médical sur l'interrogatoire d'un suspect ${ }^{3 !}$.

${ }^{30} \mathrm{CEDH} 17$ oct. 2006 Okkali c/Turquie 
Le critère principal tenant à la recherche de la manifestation de la vérité et à la mise en œuvre de diligences raisonnables, il peut donc arriver que l'article 3 ne soit pas violé alors même que l'enquête débouche sur un non-lieu. L'appréciation de l'effectivité d'une enquête relative à des allégations de mauvais traitements par des agents de l'État se fait comme toujours in concreto ${ }^{32}$.

Eu égard à cette évolution, la protection de l'article 3 a largement joué en matière garde à vue, qu'il s'agisse d'actes qualifiés de torture ou de traitements inhumains ou dégradants ${ }^{33}$ et s'est étendue aux centres de rétention administrative ${ }^{34}$, pour pénétrer de plus en plus efficacement le monde carcéral.

\section{La mise en œuvre de la notion de torture, de traitement inhumain et dégradant}

Outre les quelques cas de condamnations pour des actes de violences interindividuelles, la mise en ouvre de la notion de torture à rapidement trouvée à s'appliquer aux actes de violences commis contre les individus par des agents de l'Etat, principalement par des policiers ou des agents de l'administration pénitentiaires. Mais, de plus en plus, cet article est invoqué en matière de respect du droit à la santé des personnes privées de liberté ou au regard de leurs conditions de détention, pour ne parler que des domaines où la jurisprudence est la plus fournie.

\section{A. Le droit à la santé des détenus}

Obligation générale de soins. Le principe selon lequel la détention d'une personne malade est susceptible d'entraîner l'application de l'article 3 existe depuis longtemps dans

${ }^{31} \mathrm{CEDH} / 4$ avr. 2009, Olteanu c/Roumanie

${ }^{32} \mathrm{CEDH} 16$ juill. 20 I 5, Ghedir et autres c/ France : à propos de blessures sérieuses subies durant une garde à vue, absence de violation du volet procédural en dépit d'un non-lieu prononcé au terme de 4 ans d'instruction car dans cette affaire, la Cour a relevé, l'immédiateté de l'enquête, sa durée raisonnable, les actes dont elle témoigne (mises en examen, placement en détention provisoire, auditions de plusieurs témoins, commandement d'expertises médicales) ainsi que la constitution partie civile du requérant. L'ensemble de ces éléments ont respecté l'exigence de diligences raisonnables requise par la cour

${ }^{33}$ V. parmi la très nombreuse jurisprudence, CEDH I 8 juill. 20 I3, Nasakin c. Russie, n 22735/05 (décharges électriques).

${ }^{34} \mathrm{CEDH} 23$ juill. 20 I3, Aden Ahmed c. Malte, $\mathrm{n}^{\circ}$ 55352/I 2 (exposition potentielle des détenus au froid, absence de personnel féminin au centre de détention, absence totale d'accès à l'air libre et de périodes d'exercice pendant près de trois mois, régime alimentaire inadapté, frappant une requérante particulièrement vulnérable en raison de sa santé fragile et de son état émotionnel sur une période de quatorze mois et demi). 
la jurisprudence de la Cour européenne des droits de l'homme ${ }^{35}$. La situation des détenus est appréciée conformément aux critères généraux développés pour la mise en œuvre de l'article 3. Dès lors, pour tomber sous le coup de l'article 3, le mauvais traitement infligé doit revêtir un degré de gravité minimum et l'attitude du requérant est prise en compte pour déterminer l'issue de la requête. Mais, il ne convient pas d'opérer de distinction selon l'origine des problèmes de santé. Les détenus peuvent tirer bénéfice de l'article 3 en cas de maladie avérée ou de pathologies lourdes. Ainsi, un détenu quasiment aveugle ${ }^{36}$ ou un détenu souffrant de complications liées à une obésité de caractère héréditaire $^{37}$ sont tous deux protégés par les dispositions de cet article. L'obligation de soins en détention est générale, c'est-à-dire qu'elle s'applique sans distinction à tous les détenus et elle perdure le temps des transferts ${ }^{38}$.

Obligation de soins similaire au milieu libre. II existe donc une obligation, pour l'administration pénitentiaire, d'assurer un service de soins qui doit concerner sans exception l'ensemble de la population carcérale et dans des conditions similaires à la prise en charge en milieu libre ${ }^{39}$. A défaut, les modalités d'exécution de la peine privative de liberté pourront être considérées comme soumettant l'intéressé à une détresse ou une épreuve excédant le niveau inévitable de souffrance inhérent à la détention. II a été ainsi jugé à propos d'un refus par l'administration pénitentiaire d'une prothèse dentaire à un détenu édenté et indigent au motif qu'il ne disposait pas suffisant d'argent ${ }^{40}$.

Bien que pleinement affirmé, le principe de la protection du droit à la santé des détenus n'a connu qu'une réception mitigée, jusqu'au début des années 2000. En dépit de sa reconnaissance, fort peu d'arrêts de la Cour européenne retenaient une violation de l'article 3.

\footnotetext{
${ }^{35}$ Par ex. Comm. EDH, déc. 6 mai 1978, Kotälla c. Pays-Bas, D.R.,p. 14 ; rapp. 8 déc. 1982 , Chartier c. Italie, D.R. 33, p. 41 ; déc. 9 mai 1983, De Varga-Hirsch c. France, D.R. 33, p. I 58 ; déc. 9 mars 1988, B. c. République Fédérale d'Allemagne, D.R. 55, p. 27 I.

${ }^{36} \mathrm{Comm}$. EDH, déc. 5 oct. 1974, req. n 6181/73, Rec. 46, p. 188.

${ }^{37} \mathrm{Comm}$. EDH, Chartier c. Italie, préc.

${ }^{38} \mathrm{CEDH}$ I 4 janv. 20 I0, Moskalyuk c. Russie, n 3267/03 (l'interruption du traitement pour une tuberculose, durant une période de transfert vers une colonie correctionnelle d'une durée de 24 jours, constitue un traitement dégradant).

${ }^{39}$ Pour le CPT, « le service de santé pénitentiaire doit être en mesure d'assurer les traitements médicaux et les soins infirmiers, ainsi que les régimes alimentaires, la physiothérapie, la rééducation et toute autre prise en charge spéciale qui s'impose, dans des conditions comparables à celles dont bénéficie la population en milieu libre », Les normes du CPT, CPT/Inf/E (2002) I Rev. 2009, p. 31 .

${ }^{40} \mathrm{CEDH} 16$ févr. 2010, V.D. c. Roumanie, n 7078/02 (dans cette affaire, l'administration pénitentiaire avait mis en échec la réglementation en matière de couverture sociale des détenus, faute d'avoir signé une convention qui aurait permis de faire rembourser une partie du prix des prothèses par la caisse d'assurance à laquelle était affilié le détenu).
} 
Soins en détention ou hospitalisation. Puis, progressivement, la Cour européenne traduit le principe de protection du droit à la santé en faisant peser plusieurs obligations spécifiques sur les autorités pénitentiaires. Pour la jurisprudence, "la détention d'une personne très malade n'est pas assimilable à un traitement inhumain et dégradant si cette personne est soignée en prison et si les autorités s'engagent à l'hospitaliser en cas de besoin" ${ }^{41}$. Il en va à l'identique lorsque des détenus sont hospitalisés en vue de leur procurer un meilleur confort et un suivi régulier de leurs traitements médicaux et que le transfert n'a pas eu les effets escomptés ${ }^{42}$.

Prises de mesures particulières. Au-delà de la double contrainte imposant de fournir des soins en détention ou d'hospitaliser les détenus, les autorités pénitentiaires sont tenues de se soumettre à d'autres obligations, exposées dans un second temps par la Cour. Elles doivent également au regard de la maladie du sujet adopter "des mesures particulières". II peut s'agir bien évidemment d'une hospitalisation, "mais aussi de tout autre placement dans un lieu où le condamné malade aurait été suivi et sous surveillance, en particulier la nuit ${ }^{143}$.

Cette obligation tient compte du fait que tous les détenus malades n'ont pas nécessairement à être envoyés dans un hôpital alors même qu'ils peuvent nécessiter un suivi médical et une surveillance qu'il est impossible d'apporter en prison, surtout la nuit. Elle peut dès lors se traduire pour l'Etat par une obligation d'un suivi spécialisé dans une structure adaptée que requiert l'état du requérant ${ }^{44}$ ainsi par la prévision de solutions alternatives à l'hospitalisation pour les détenus malades (par ex. suspension de peine notamment pour les détenus très âgés et/ou malades même si le pronostic vital n'est pas forcément engagé, placement sous surveillance électronique, placement en résidence ou en établissement médical surveillé... $)^{45}$.

Lorsque la situation personnelle du détenu le commande, l'état doit donc tout mettre en œuvre pour éviter un maintien en détention, y compris de façon transitoire dans l'attente d'une solution pérenne. ${ }^{46}$ Dès lors, dans des cas exceptionnels où l'état de

\footnotetext{
${ }^{41}$ Par ex. Comm. EDH, 28 janv. 1994, Hurtado c. Suisse, Série A, n 280-A, §§ 79-80.

${ }^{42} \mathrm{CEDH} 9$ juill. 20 I 5, Martzaklis et autres c. Grèce, $n^{\circ}$ 20378/I3 (transfert de détenus séropositifs dans un hôpital pénitentiaire).

${ }^{43}$ CEDH 14 nov. 2002, Mouisel c. France, RTDH 2003, p. 999, note J.P. Céré.

${ }^{44} \mathrm{CEDH} 2$ I déc. 20 I0, Taddei c. France, n³6435/07, AJ Pénal 201 I , p. 129, note J.P. Céré.

${ }^{45} \mathrm{CEDH} 12$ janv. 2010 , Schwartz c. Roumanie, $n^{\circ} 28304 / 02$.

${ }^{46} \mathrm{CEDH} 17$ juill. 2012, Scoppola c. Italie ( $n^{\circ} 4$ ), $n^{\circ}$ 65050/09 (traitement inhumain et dégradant pour avoir maintenu un requérant âgé de 72 ans ne pouvant se déplacer qu'en fauteuil roulant et souffrant de pathologies cardiaques et du métabolisme, de diabète, d'un affaiblissement de sa masse musculaire empêchant la position assise, d'hypertrophie de la prostate et de dépression durant plusieurs mois dans un établissement pénitentiaire, en dépit des avis des experts et du juge de l'application des peines, dans l'attente d'une mesure de suspension d'exécution de la peine.
} 
santé du détenu est absolument incompatible avec sa détention, l'article 3 peut exiger la libération de la personne concernée sous certaines conditions ${ }^{47}$ et cette sortie de prison doit intervenir dans des délais appropriés. ${ }^{48}$

Droit de recours. II ressort, par ailleurs, de la jurisprudence que le détenu doit bénéficier d'un droit de recours lui permettant de contester la pertinence d'un maintien en détention à l'aune de son état de santé. Les " procédures judiciaires peuvent être susceptibles de constituer des garanties pour assurer la protection de la santé et du bien être des prisonniers que les Etats doivent concilier avec les exigences légitimes de la peine privative de liberté ${ }^{49}$.

Effectivité, adéquation et transparence des soins. Un suivi médical doit être proposé à tout détenu mais le respect de cette seule exigence est en réalité insuffisant pour affranchir les autorités pénitentiaires d'un constat d'inconventionalité. La réponse médicale doit être effective, adéquate et transparente ${ }^{50}$. La contrainte sanitaire qui en découle doit s'insérer dans une stratégie thérapeutique globale et ne pas se cantonner à la lutte contre les seuls symptômes rencontrés ${ }^{51}$. Dès lors, une absence de tout traitement ${ }^{52}$, une réponse médicale irrégulière ${ }^{53}$, tardive ${ }^{54}$ ou inadapté $e^{55}$, un constat de défaillance ${ }^{56}$ ou un diagnostic trop tardif de la maladie du détenu amène une violation de

${ }^{47}$ CEDH I 9 juill. 2007, Rojkov c. Russie, no 64 I 40/00, § 104

${ }^{48} \mathrm{CEDH}$ I I févr. 20 I 4, Contrada $\left(\mathrm{n}^{\circ} 2\right)$ c. Italie, $\mathrm{n}^{\circ} 7509 / 08$ (détention à domicile intervenue neuf mois après la première demande).

${ }^{49}$ CEDH 14 nov. 2002, Mouisel c. France, § 44 ; RTDH 2003, p. 999, note J.P. Céré.

${ }^{50} \mathrm{CEDH} 27$ nov. 20 I2, Dirdizov c. Russie, n 4| 46 I/I0 ; CEDH 9 janv. 20 I 4, Budanov c. Russie, $\mathrm{n}^{\circ} 66583 / \mathrm{ll}$.

${ }^{51} \mathrm{CEDH} 29$ nov. 2007, Hummatov c. Azerbaïdjan, n 9852/03 et I 3413/04

${ }^{52} \mathrm{CEDH}$ I 4 oct. 201 I , Logvinenko c. Ukraine, $\mathrm{n}^{\circ}$ I 3448/07 (traitement inhumain et dégradant en l'absence de surveillance médicale étroite et de traitement pour la tuberculose et le virus $\mathrm{HIV}$ ); ${ }^{5050}$ CEDH I 4 oct. 201 I , A. B. c. Russie, $n^{\circ}$ I 439/06 (traitement inhumain et dégradant en l'absence de surveillance médicale minimum et de traitement antiviral contre le virus HIV).

${ }^{53} \mathrm{CEDH}$ I 3 nov. 20 I 2, Koryak c. Russie, $\mathrm{n}^{\circ}$ 24677/I 0 (interruption de traitement contre le VIH et la tuberculose).

${ }^{54} \mathrm{CEDH} 22$ avril 20 I4, G.C. c. Italie, $\mathrm{n}^{\circ} 73869 / \mathrm{l} 0$ (incontinence faisant suite à une opération des hémorroïdes).

${ }^{55} \mathrm{CEDH} 3$ I mars 20 I 5, Davtyan c. Arménie, n 29736/06 (traitement symptomatique au lieu d'un traitement spécialisé) ; CEDH 5 déc. 2013, Kutepov c. Russie, n 13 182/04 (réponse médicale inadaptée au traitement d'une myélopathie); $\mathrm{CEDH} 2$ févr. 2016, Dr gan c. Roumanie, $n^{\circ}$ 65 158/09 (régime alimentaire inadapté à des problèmes dentaires liés à une parodontite).

${ }^{56}$ Le Comité de prévention contre la torture est à l'unisson en considérant également qu'« un niveau de soins médicaux insuffisant peut conduire rapidement à des situations qui s'apparentent à des traitements inhumains ou dégradants », Les normes du CPT, CPT/Inf/E (2002) I Rev. 2009, p. 29. 
l'article 3 de la Convention ${ }^{57}$. L'obligation médicale de prise en charge s'entend également de soins paramédicaux ${ }^{58}$. Un suivi médical peut s'imposer, indépendamment de toute affection, compte tenu de l'état de vulnérabilité du sujet ${ }^{59}$.

La jurisprudence laisse cependant une marge de manœuvre aux autorités dans le choix du traitement à opérer entre les différentes catégories de traitement appropriées aux pathologies des détenus. Elle recherche donc si l'état de santé de l'individu et le traitement administré ont été correctement évalués ${ }^{60}$.

Attitude du détenu. Conformément une fois encore à sa jurisprudence générale, la violation de l'article 3 ne peut être retenue que si le mauvais traitement infligé atteint un degré de gravité minimum et sous réserve de l'attitude du requérant. Dans l'hypothèse où le détenu refuse de se faire soigner ou s'il participe à la dégradation de son état de santé, il ne saurait y avoir de violation de l'article ${ }^{61}$. Ce constat est cependant parfois tempéré. Ainsi une violation de l'article 3 est a-t-elle pu être admise lorsque le refus de soins décidé par le détenu était initié par l'attitude des autorités pénitentiaires ou policières ${ }^{62}$ ou encore quand le refus du requérant d'être transféré dans un hôpital civil n'avait été envisagé qu'à titre provisoire dans l'attende d'une solution définitive convenable ${ }^{63}$.

${ }^{57}$ CEDH 5 avr. 20I I, Vasyukov c. Russie, $n^{\circ} 2974 / 05$.

${ }^{58} \mathrm{CEDH} 15$ févr. 20 I 5, Helhal c. France, n ${ }^{\circ} 10401 / 12$, Al Pénal 20 I5, p. 219 , obs. J.P. Céré ; D. 2015. I 122, obs. Céré ; AJDA 20 I5, p. 1732, obs. L. Burgorgue-Larsen (absence de séances de kinésithérapie pour un détenu incontinent).

${ }^{59}$ CEDH 24 mars 20 I6, Korneykova et Korneykov c. Ukraine, n 56660/I2 (absence de suivi durant 3 mois et demi environ d'un nouveau né)

${ }^{60} \mathrm{CEDH}$ I sept. 2016, Wenner c. Allemagne, $\mathrm{n}^{\circ}$ 62303/I3 (absence d'étude avec soin de la thérapie devant convenir à un détenu héroïnomane de longue date).

${ }^{61} \mathrm{CEDH} 23$ févr. 20 10, Dermanović c. Serbie, n 48497/06 (détenu qui retarde le diagnostic en entamant une grève de la faim et en refusant des examens à l'hôpital).

${ }^{62}$ Par ex. Cour EDH, I 4 nov. 2002, Mouisel c. France, JCP 200 I. I. I09, obs. F. Sudre ; D. 203, p. 524, obs. JF. Renucci ; Rev. sc. crim. 2003, p. I 44, obs. F. Massias ; Dr. pénal 2003, comm. 52, obs. A. Maron et M. Haas; RTDH, 2003, p. 999, noteJ.P. Céré.

${ }^{63} \mathrm{CEDH} 17$ juill. 20 I2, Scoppola c. Italie ( $\left.n^{\circ} 4\right), n^{\circ}$ 65050/09. La Cour ajoute qu'il est difficile de concevoir que ce refus ait été en mesure, en lui-même, d'entraver les efforts des autorités de trouver une structure adéquate. 
Pour la Cour, le maintien en détention de personnes dont la pathologie est durablement incompatible avec le maintien en détention est assurément de nature à violer l'article 3. La protection conventionnelle doit s'appliquer indépendamment du statut juridique de l'individu et recouvre aussi bien la période de détention provisoire que le temps de la condamnation. Ainsi le refus d'une demande de remise en liberté d'un prévenu inapte à demeurer incarcéré, au motif que les seuls bénéficiaires de la procédure protégeant la santé des détenus atteints de maladie grave doivent avoir fait l'objet d'une condamnation définitive $e^{64}$.

Les détenus handicapés. II est acquis que le maintien en détention d'une personne handicapée doit être compatible avec son handicap, indépendamment de l'existence d'une intention d'humilier ou de rabaisser la personne concernée. La cour européenne considère que le placement ou le maintien en détention d'une personne handicapée oblige les autorités à veiller avec une vigilance particulière à ce que les conditions de sa détention s'accordent aux besoins spécifiques de son infirmité ${ }^{65}$.

Les détenus atteints de troubles psychiatriques. La détention de personnes souffrant de troubles mentaux est également susceptible de poser des difficultés sous l'angle de l'article 3, notamment une incarcération dans un établissement pénitentiaire non prévu pour accueillir de telles personnes ${ }^{66}$. II n'est donc pas concevable de traiter sur le même pied que les autres détenus, un individu atteint de troubles psychiatriques ${ }^{67}$. Pour ce public, il convient donc, plus particulièrement, de « tenir compte de leur vulnérabilité et de leur incapacité, dans certains cas, à se plaindre de manière cohérente ou à se plaindre tout court des effets d'un traitement donné sur leur personne ${ }^{68}$ ".

\section{B. Les conditions de détention}

Absence de volonté d'humiliation. La protection de l'article 3 sur le terrain des conditions de détention joue indépendamment de toute volonté d'humiliation du détenu. Au terme d'une évolution de la jurisprudence engagée au début des années

\footnotetext{
${ }^{64}$ CEDH 5 mars 2013, Gülay Çetin c. Turquie, n 44084/I0 (le maintien en détention d'une personne atteinte d'un cancer avancé est constitutif d'un traitement inhumain et dégradant).

${ }^{65} \mathrm{CEDH}$ I 0 juill. 200 I, Price c. Royaume-Uni, n 33394/96, AJDA 200 I. I060, chron. J.-F. Flauss ; Rev. sc. crim. 200 I , p. 88I , obs. F. Tulkens ; JCP G 2002. I. I05, obs. F. Sudre ; CEDH I 2 mars 20।3, Zarzyckic. Pologne, n I535।/03.

${ }^{66} \mathrm{CEDH}$, Aerts c. Belgique, préc. ; Cour EDH 3 avr. 200 I, Keenan c. Royaume-Uni, D. 2002, p. I 18 , obs. J.P. Céré.

${ }^{67}$ CEDH 18 déc. 2007, Dybeku c. Albanie, n 4II53/06 (détenu atteint de schizophrénie paranoïaque chronique qui partageait sa cellule avec des détenus en bonne santé et qui était traité comme un détenu ordinaire).

${ }^{68}$ Cour EDH, Aerts c. Belgique, préc. § 66 ; Keenan c. Royaume-Uni, préc. § I I I ; CEDH 20 janv. 2009, Musial c. Pologne, $n^{\circ}$ 28300/06.
} 
2000, le constat de conditions de vies déficientes suffit à générer une violation de l'article 3 , principalement lorsqu'il s'agit de surpopulation chronique et de défaillance en matière de respect des règles d'hygiène ${ }^{69}$.

Indifférence du lieu de détention. Au regard des décisions rendues par la cour, il est possible de préciser que la protection recouvre tous les lieux de détention, ce qui signifie qu'un Etat peut être condamné, indépendamment même de toute surpopulation, à raison d'un seul constat de mauvaises conditions de détention. Ainsi, il a été jugé que l'article 3 était invocable pour des détenus séjournant dans un quartier de haute sécurité ${ }^{70}$ ou dans un couloir de la mort ${ }^{71}$, dans une cellule disciplinaire ${ }^{72}$, dans une cellule d'isolement $^{73}$, dans un hôpital pénitentiaire ${ }^{74}$, dans des locaux de police ${ }^{75}$, dans des postes frontières $^{76}$ ou encore dans un centre de rétention pour étranger ${ }^{77}$. Les conditions de détention pourront donc conduire à une violation de l'article 3 à l'aune de leurs effets cumulatifs sur le détenu ${ }^{78}$.

${ }^{69} \mathrm{~V}$. pour les premières décisions de condamnation, CEDH I 5 juill. 2002, Kalachnikov c. Russie, Rec. 2002-VI (cellule prévue pour 8 personnes et I 8 à 24 détenus présents, un lit à partager entre 2 ou 3 détenus, lumière constamment allumée, aération déficiente de la cellule alors que certains détenus fumaient, présence de rats ; le détenu avait été incarcéré pendant 5 ans dans de telles conditions); CEDH 13 sept. 2005, Ostrovar c. Moldavie, AJP 2005, p. 42 I , obs. J.P. Céré.

${ }^{70}$ CEDH 4 févr. 2003, Lorsé et autres c. Pays-Bas et CEDH 4 févr. 2003, Van der Ven c. Pays-Bas, JCP 2003. I. I60, n² 2, obs. F. Sudre; Rev. sc. crim. 2004. 44I , obs. F. Massias

${ }^{71}$ CEDH 8 juill. 2004, llascu et autres c/Moldova et Russie, n 48787/99.

${ }^{72} \mathrm{CEDH} 20$ janv. 201 I, Payet c. France, D. 201 I , p. 643, note J.P. Céré ; AJ Pénal 201 I , p. 88, obs. M. Herzog-Evans, AJDA 200 I , 1997, obs. L. Burgorgue-Larsen ; CEDH I 0 nov. 201 I , Plathey c. France, $n^{\circ} 48337 / 09$.

${ }^{73}$ CEDH 5 mars 2013, Geanopol c. Roumanie, $n^{\circ}$ 1777/06 (détenu sous traitement psychiatrique).

${ }^{74} \mathrm{CEDH}$ I 8 déc. 2012, Čuprakovs c. Lettonie, $n^{\circ} 8543 / 04$

${ }^{75} \mathrm{CEDH} 25$ oct. 201 I, Ibram c. Grèce, n ${ }^{\circ}$ 39606/09 (période de détention provisoire de quatre mois dans des locaux inadaptés, destinés à accueillir des personnes pour une courte durée). Adde pour une période de trois mois, CEDH 9 oct. 20 I2, Dimopoulos c. Grèce, n 49658/09; période de détention de 53 jours, CEDH I I juin 20 I3, Marin Vasilescu c. Roumanie, n62353/09.

${ }^{76} \mathrm{CEDH}$ II déc. 20 I4, Al.K. c. Grèce, n 63542/I I ; CEDH I I juin 2009, S.D. c. Grèce, n $5354 \mathrm{I} / 07$.

${ }^{77} \mathrm{CEDH} 26$ juin 20 I4, De los Santos et de la Cruz c. Grèce, n ${ }^{\circ} 2134 / 12$ et $2161 / 12$; CEDH 24 juin 20 I4, Yarashonen c. Turquie, $n^{\circ} 727$ I0/I I ; CEDH 12 déc. 2013, Khuroshvili c. Grèce, $n^{\circ}$ $58165 / 10$.

${ }^{78} \mathrm{CEDH} 28$ mai 20 13, Sabev c. Bulgarie, n²7887/06 (régime spécial d'isolement durant près de 8 ans durant lequel le détenu était contraint d'utiliser un sceau pour faire ses besoins, faute de toilettes dans la cellule). 


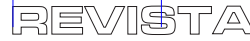

Locaux de police et la nécessaire adaptation à la durée de détention. Un critère d'adaptation du lieu de détention à la durée de la détention doit exister. La cour estime que de par leur nature même, les locaux de police sont des lieux destinés à accueillir des personnes pour une courte durée ${ }^{79}$. II en résulte que indépendamment des déficiences en termes de conditions de détention générées par le surpeuplement, les locaux de police ne sont pas considérés comme adaptés aux besoins d'une incarcération prolongée de personne en détention provisoire ou incarcérées en vue de leur expulsion ${ }^{80}$.

Conditions de transport. Tel est le cas encore lors de trajets imposés, comme à l'occasion de différents transferts, pour un détenu placé à l'isolement pendant 5 à 8 heures et qui disposait de moins de 1,5 m2 d'espace personnel ${ }^{81}$ ou encore pour un détenu lors de trajets entre l'établissement et le tribunal qui pouvaient durer plusieurs heures $^{82}$ ainsi qu'a fortiori pour un cumul de mauvaise conditions de transport et de détention dans les cellules du tribunal ${ }^{83}$. La cour considère que des conditions de transports exécrables excèdent le seuil de gravité de l'article 3 car elles nuisent notamment concentration et à la vigilance particulièrement nécessaire de l'intéressé au cours de son procès ${ }^{84}$. La violation est également encourue lorsque les détenus ne bénéficient pas de lumière et qu'ils sont susceptibles de perdre l'équilibre durant le transport ${ }^{85}$.

${ }^{79}$ CEDH 27 juill. 2006, Kaja c. Grèce, n 32927/03 ; CEDH 21 juin 201 I, Efremidze c. Grèce, n ${ }^{\circ}$ $33225 / 08$

${ }^{80} \mathrm{~V}$. pour des durées de détention de un à trois mois, CEDH 17 avril 2014, Kavouris et autres C. Grèce, $n^{\circ} 73237 / 12$; CEDH 17 avr. 20 I4, Adamantidis c. Grèce, n 10587/I0 ; CEDH 21 juin 201 I , Efremidze c. Grèce, ibid. ; CEDH 29 oct. 2009, Shuvaev c. Grèce, n 8249/07.

${ }^{81} \mathrm{CEDH}$ ler déc. 2009, Jeronovics c. Lettonie, n 547/02.

${ }^{82} \mathrm{CEDH} 22$ mai 20 I2, Idalov c. Russie, n ${ }^{\circ}$ 5826/03 (violation de l'article 3 sur les seules conditions de transport dans des fourgons d'une hauteur d' I m60 environ, avec une surface de compartiment entre 8 et I I m2, qui transportaient de 25 à 36 personnes).

${ }^{83} \mathrm{CEDH} 9$ janv. 2013, Retunscaia c. Roumanie, $n^{\circ} 2525$ I/04, (fourgon servant aux trajets entre le tribunal et la prison surpeuplé cumulé avec des cellules du tribunal sales et non dotées d'aération).

${ }^{84} \mathrm{CEDH} 6$ nov. 20 12, Maksim Petrov c. Russie, $n^{\circ} 23185 / 03$ (trente deux déplacements sur un an et deux mois dans des camions qui contenaient deux fois plus individus que la capacité de conception). $V$. aussi, indépendamment des conditions de détention, pour un requérant privé de nourriture et de sommeil les jours où il était emmené au tribunal, CEDH 6 nov. 20 I2, Strelets C. Russie, $n^{\circ} 28018 / 05$.

${ }^{85} \mathrm{CEDH} 10$ juin 20 I4, Voicu c. Roumanie, $\mathrm{n}^{\circ} 22015 / 10$. 
Toutefois l'examen de la jurisprudence de la cour européenne permet de dégager un critère purement factuel tenant justement à la surface disponible. $3 \mathrm{~m} 2$ apparaît comme un standard minimum en deçà duquel une violation de l'article 3 est inévitable ${ }^{86}$ tandis qu'un espace personnel supérieur à $4 \mathrm{~m} 2$ est considéré comme suffisant ${ }^{87}$.

Surpopulation sévère. La présence d'un espace de vie en cellule particulièrement restreint suffit donc à lui seul pour conclure violation de l'article 3. Lorsque la surpopulation carcérale atteint un certain niveau, " le manque d'espace dans un établissement pénitentiaire peut constituer l'élément central à prendre en compte dans l'appréciation de la conformité d'une situation donnée à l'article $3{ }^{88}$. Cela concerne les cas de surpopulation flagrante quand l'espace personnel accordé au détenu est inférieur à $3 \mathrm{~m}^{2^{89}}$, quelque soit la superficie exacte ${ }^{90}$. Le critère dégagé par la cour pour apprécier le manque d'espace est triple: " chaque détenu doit disposer d'une possibilité de couchage individuel dans la cellule ; chaque détenu doit disposer d'au moins $3 \mathrm{~m}^{2}$ d'espace au sol ; et la surface globale de la cellule doit être telle qu'elle autorise les détenus à circuler librement entre les meubles »". Elle estime alors que l'Etat, "par le

${ }^{86}$ CEDH 12 juill. 20 I2, Vartic c. Roumanie, $n^{\circ}$ I 1 52/05 ; CEDH 16 juill. 2009, Marian Stoicescu c. Roumanie, $n^{\circ}$ 12934/02 ; CEDH 22 oct. 2009, Orchowski c. Poland, $n^{\circ}$ 17885/04 ; CEDH 6 déc. 2007, Lind c. Russia, n² 25664/05; CEDH 21 juin 2007, Kantyrev v. Russia, n 37213/02 ; CEDH I 6 juin 2005, Labzov v. Russia, n 62208/00.

${ }^{87} \mathrm{CEDH} 12$ déc. 2013, Kanakis c. Grèce $\left(n^{\circ} 2\right), n^{\circ} 40146 / 1$ I (4,8 m2)

${ }^{88} \mathrm{CEDH} 7$ avr. 2005, Karalevičius c. Lituanie, no 53254/99; CEDH 2 nov. 2010 , Grozavu c. Roumanie, no 24419/04; CEDH 5 juin 20 I2, Ciuc c. Roumanie, n³4485/09.

${ }^{89}$ Par ex. CEDH 22 juill. 20।4, Bulatović c. Monténégro, n 67320/I0 (2,8 m2); CEDH I 9 déc. 2013, Tunis c. Estonie, $n^{\circ} 429 / 12$ (2,38 à 2,55 m2); CEDH 2 juill. 20।3, Fehér c. Hongrie, $n^{\circ}$ 69095/I 0 (2, I 6 à 2,75 m2); CEDH 4 déc. 20 I2, Leontiuc c. Roumanie, n 44302/I 0 (1,04 à I,77 m2) ; CEDH 13 nov. 20 12, Constantin Modarca c. République de Moldavie, n 37829/08 ( I,5 I à 2,5 m2); CEDH 30 oct. 20 I 2, Ardelean c. Roumanie, $n^{\circ}$ 28766/04 (I ,66 m2 et 2,59 m2); CEDH 23 oct. 20 I2, Dmitriy Rozhin c. Russie, $n^{\circ}$ 4265/06 (2,3 m2 et certains jours I, I I m2); CEDH 17 juill. 20 I2, Budacca c. Roumanie, $n^{\circ}$ 5760/I0 (I,44 m2); CEDH 26 juin 20 I2, Petrea Chis I u c. Roumanie, n 36680/03 (I,22 m2). CEDH 20 oct. 20 I I, Mandic et Jovic c. Slovénie, $n^{\circ}$ 5774/I 0 et 5985/I0 ; CEDH 24 juin 20 I0, Veliyev c. Russie, $n^{\circ}$ 24202/05; CEDH I er juill. 2010 , Daydov et autres c. Ukraine, n० 17674/02 ; Kantyrev c. Russie, no 372।3/02, §§ 50-5।, 21 juin 2007, Frolov c. Russie, no 205/02, §§ 47-49, 29 mars 2007, Kadikis c. Lituanie, no 62393/00, $\S 55,4$ mai 2006, et Melnik c. Ukraine, no 72286/0 I § I02, 28 mars 2006 ; CEDH 9 oct. 20 I2, Asyanov c. Russie, $n^{\circ}$ 25462/09 (2,8 m2).

${ }^{90} \mathrm{CEDH} 28$ mai 20 I 4, Tsokas et autres c. Grèce, $n^{\circ} 4 / 513 / 12$.

${ }^{91} \mathrm{CEDH} / 2$ mars 2015, Muršićc. Croatie, n7334/I3. 


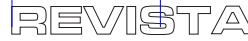

biais de ses organes spécialisés, n'a pas déployé tous les efforts nécessaires afin d'assurer au requérant des conditions de détention qui soient compatibles avec le respect de la dignité humaine et afin d'assurer que les modalités d'exécution de la mesure ne soumettent pas l'intéressé à une détresse ou à une épreuve d'une intensité qui excède le niveau inévitable de souffrance inhérent à la détention ${ }^{92} »$. Dans ce cas, le constat de violation de l'article 3 est suffisamment opérant à lui seul pour que la Cour ne juge pas nécessaire d'examiner d'autres critères concernant les conditions matérielles de détention ${ }^{93}$.

Renversement de la présomption de traitement inhumain ou dégradant. L'établissement d'un constat selon lequel un détenu dispose de moins de 3 mètres carrés d'espace au sol entraîne une présomption forte selon laquelle les conditions de détention équivalent à un traitement contraire à l'article 3.

La Cour va donc nécessairement tenir compte du contexte environnant. Celui-ci peut conduire à une appréciation de traitement inhumain et dégradant ${ }^{94}$, notamment quand les locaux sont destinés à une détention de courte durée ${ }^{95}$. Et, dans l'hypothèse de surpopulation avérée, des conditions d'hygiène tout à fait acceptables ne suffisent pas à éviter un constat de violation de l'article $3^{96}$ ni le fait que le détenu dispose d'une télévision, d'une radio et qu'il ait accès à de nombreux périodiques ou livres est inopérant ${ }^{97}$, ni le fait d'invoquer un temps d'accès allongé à des espaces commun ${ }^{98}$ ou encore d'indiquer que le détenu bénéficiait durant certaines périodes d'un espace supérieurà $4 \mathrm{~m} 2^{99}$.

\footnotetext{
${ }^{92} \mathrm{CEDH} 17$ juill. 2012, Budacca c. Roumanie, ibid. ; CEDH I I déc. 2012, Banu c. Roumanie, $\mathrm{n}^{\circ}$ 60732/09.

${ }^{93}$ Par ex. CEDH 28 oct. 20 I4, Tirean c. Roumanie, $n^{\circ}$ 47603/I0, § 46.

${ }^{94}$ Ex. CEDH 24 nov. 201।, Tsygoniy c. Ukraine, n 192213/04 (manque d'hygiène et absence quotidienne d'exercice).

${ }^{95} \mathrm{CEDH} 15$ janv. 2013, Velichko c. Russie, n० $19664 / 07$ (détention pendant 8 mois dans une cellule d'un centre de détention prévue pour un accueil limité à dix jours ne comportant ni toilette ni fenêtre ni l'accès à la lumière ou à l'air naturel).

${ }^{96} \mathrm{CEDH} 22$ oct. 2009, Sikorski c. Pologne, $\mathrm{n}^{\circ} 17599 / 05$.

${ }^{97} \mathrm{CEDH} 26$ mai 2009, Maciuca c. Roumanie, n² 25763/03.

${ }^{98} \mathrm{CEDH} 26$ mars 20 I3, Györgypál c. Roumanie, $n^{\circ}$ 29540/08 (placement en régime semi-ouvert, avec ouverture des portes des cellules et accès au cours de promenade porté à 7 h45 par jour. $\mathrm{V}$. aussi CEDH 27 mai 201 0, Ogica c. Roumanie, n²4708/03, Dr. pén. avr. 20 I I, p. 2 I , chron. E. Dreyer; CEDH IO juin 20 I 4, Bujorean c. Roumanie, $n^{\circ}$ I3054/I 2

${ }^{99} \mathrm{CEDH} 28$ oct. 2014 , Tirean c. Roumanie, $\mathrm{n}^{\circ}$ 47603/I 0
} 
Surpopulation. Lorsque le manque d'espace est moins sévère (entre 3 et $4 \mathrm{~m} 2$ ) et que la surpopulation n'est pas importante au point de soulever, pour la Cour, à elle seule un problème sous l'angle de l'article 3 , cette dernière intègre d'autres aspects des conditions matérielles de détention pour apprécier la conformité d'une situation donnée à l'article 3 de la Convention. C'est le cas pour des éléments tels que la possibilité pour un requérant de bénéficier d'un accès aux toilettes dans des conditions respectueuses de son intimité, la ventilation, l'accès à la lumière naturelle, l'état des appareils de chauffage ainsi que la conformité avec les normes d'hygiène.

L'exiguïté combinée avec l'un des facteurs suivants entraîne ainsi une violation de l'article 3:

- impossibilité de bénéficier d'une douche plus d'une fois par semaine ${ }^{100}$,

- absence de ventilation dans la cellule ${ }^{101}$,

- absence de lumière naturelle dans la cellule ${ }^{102}$,

- manque de facilités sanitaires ${ }^{103}$,

- exercice en plein air d'une durée très limitée ${ }^{104}$

- absence totale d'intimité ${ }^{105}$

Au terme d'un examen de la jurisprudence de la cour européenne des droits de I'homme sur son interprétation de la notion de torture, de traitements, inhumain set dégradants qui n'avait pas la prétention à l'exhaustivité, vous avez, je l'espère, pu vous convaincre, de l'importance de cette jurisprudence. Celle-ci a permis de de nombreux Etats en Europe d'avancer vers un chemin plus vertueux en permettant de limiter les actes accomplis contre l'intégrité physique ou morale des citoyens, y compris pour les victimes qui subissent une privation de liberté, quelle que soit sa forme ou sa nature. Au surplus, la cour européenne des droits de l'homme a construit au fil du temps une

\footnotetext{
${ }^{100} \mathrm{CEDH} 22$ avr. 2010 , Goroshchenya c. Russie, n 387। I/0, § 71.

${ }^{101} \mathrm{CEDH} 20$ mai 2010 , Viloguzov c. Ukraine, $\mathrm{n}^{\circ} 32362 / 02$, $\$ 60$.

${ }^{102} \mathrm{CEDH} 22$ mai 20 I0, Goroshchenya c. Russie, $n^{\circ} 387$ I I/0, § 7 I

${ }^{103} \mathrm{CEDH} 6$ déc. 2007, Bragadireanu c. Roumanie, $n^{\circ} 22088 / 04$ (détenu ayant un anus artificiel qui devait s'en remettre à ses codétenus pour satisfaire ses besoins naturels, n'avait pas de possibilité de se doucher et ne disposait pas d'eau chaude).

${ }^{104} \mathrm{CEDH} 17$ janv. 20 I2, István Gábor Kovács C. Hongrie, n 1 5707/I0 ; CEDH 27 janv. 20 I I, Yevgeniy Alekseyenko c. Russie, n $41833 / 04$; CEDH 21 déc. 2010, Gladkiy c. Russie, n 3242/03.

${ }^{105}$ CEDH ler mars 2007, Belevitskiy c. Russie, n 72697, §§ 73-79; CEDH 2 juin 2005, Novoselov c. Russie, n 66460/0 I, §§ 32 et 40-43.
} 
jurisprudence qui permet de sanctionner efficacement de tels actes lorsqu'ils sont avérés et ce n'est pas le moindre des atouts de cette jurisprudence. II ne faut pas se voiler la face, sans la pression de la cour européenne, nombre de pays et je pense aussi à France, n'aurait jamais cherché à combattre ce fléau indigne d'un pays démocratique.

\section{REFERENCES}

Les normes du CPT, CPT/Inf/E(2002) . I Rev. 2009, p. 29.

TULKENS, V. F. Droits de l'homme et prison in J.P. Céré (dir.), Panorama européen de la prison, L'Harmattan, 2002, p. 35.

Recebido em: |4/II/2016

Aprovado em: 21/12/2016 